\title{
Effect of Knee Valgus Angle during Single Leg Squat and Horizontal Hop for Distance in Patients with Patellofemoral Pain and Controls
}

\author{
Hussain S. Ghulam \\ Department of Physical Therapy, Faculty of Applied Medical Science, Najran University, Najran, Saudi Arabia \\ Email: hsghulam@nu.edu.sa
}

How to cite this paper: Ghulam, H.S. (2021) Effect of Knee Valgus Angle during Single Leg Squat and Horizontal Hop for Distance in Patients with Patellofemoral Pain and Controls. International Journal of Clinical Medicine, 12, 261-271.

https://doi.org/10.4236/ijcm.2021.126023

Received: May 14, 2021

Accepted: June 14, 2021

Published: June 17, 2021

Copyright $\odot 2021$ by author(s) and Scientific Research Publishing Inc. This work is licensed under the Creative Commons Attribution International License (CC BY 4.0).

http://creativecommons.org/licenses/by/4.0/

\begin{abstract}
Background: Patellofemoral pain (PFP) is considered one of the most common dysfunctions of the lower extremities. Faulty lower limb mechanics and increased of knee valgus on loaded tasks are believed to play an important role in the development of PFP. Objective: To figure out if male PFP patients during single leg horizontal hop for distance and squat with greater knee valgus than controls, and if the nature of the task changes the angles of knee valgus. Methods: Twenty males with unilateral PFP formed the patient group and forty-five asymptomatic males formed the control group. Two dimensional (2-D) frontal plane projection angle (FPPA) was used during single leg squatting and horizontal hop for distance tasks. Results: For the single leg squat, the mean of $6.96^{\circ}, 9.80^{\circ}, 15.04^{\circ}$ was reported in the control, PFP asymptomatic knee, and PFP symptomatic knee, respectively. For the single leg horizontal hop for distance, the mean of $11.63^{\circ}, 13.72^{\circ}, 19.17^{\circ}$ was reported in the control, PFP asymptomatic knee, and PFP symptomatic knee, respectively. These differences were significant $(p<0.002)$ for both tasks. Conclusions: Patients with PFP represented with greater knee valgus angle than what was found in either their asymptomatic limb or in the control group.
\end{abstract}

\section{Keywords}

Knee Valgus, Hop Tests, Squat, Patellofemoral Pain, Controls

\section{Introduction}

Patellofemoral pain (PFP) is one of the most common dysfunctions and disorders of lower extremities, mainly affecting young physically active athletes [1]. The presence of PFP usually limits participation in sporting activities [2]. This 
disorder has been reported to develop patellofemoral osteoarthritis [1] [3]. The mechanisms are still not clearly understood, however, faulty lower limb mechanics and increased of knee valgus on loaded tasks are believed to play an important role in the development of PFP [4].

As the patella passes through the trochlear groove, it has been thought that abnormality in lower limb biomechanics is claimed to negatively affect the alignment of the patella [5]. Wilson et al. [5] reported that PFP patients had increased lateral patellar subluxation and tilting during squatting with the neutral aligned position knee. Tanamas et al. [6] found that increased lateral patellar tilt being correlated with both increased stress on loading and decreased medial and lateral patella facet cartilage volumes. Noehren et al. [7] found a significant relationship between lateral patella translation and knee abduction and external rotation when asymptomatic participants squatted with knees aligned in a valgus or neutral position. Abnormal distribution of the stresses on the patellofemoral joint will happen when the load-bearing surface areas are changed, with different patellar tracking [8]. This abnormal distribution of stresses is believed to have a strong relationship with patellar dysfunctions such as osteoarthritis [9]. Lee [10] reported that patients with PFP showed significant greater knee valgus angle on the affected limb during loading tasks than that reported in either their sound limb or in the asymptomatic control group.

In clinical research, two-dimensional (2-D) motion-analysis system is used to measure different functional movement tasks and can be easily found in clinical practice. 2-D motion-analysis system has been used in the literature to evaluate dynamic knee valgus in many screening tests [10] [11] [12] [13]. These tests involved the single leg squat [10], drop vertical jump [11], single-leg horizontal hop for distance [12], and drop landing [13]. Moreover, 2-D analysis has been used to evaluate knee-valgus angle in healthy, athletic, and injured populations [11].

Poor limb alignment, especially an increased knee valgus during single leg squat [14], running [15], and bilateral landing tasks [16], has been correlated with PFP. Therefore, one of the mentioned studies [10] investigated different movement patterns happened in different single leg movement tasks and how they link to the presence of PFP in female patients. However, none of the above-mentioned studies investigated the changes happened in knee valgus angle between different single leg movement tasks in male participants and how that might relate to the presence of PFP, especially when there are differences in knee valgus angles between gender according to the body constitution. Therefore, the aim of this study is to evaluate the knee valgus angle of male PFP patients and asymptomatic controls while undertaking two tasks, single leg horizontal hop for distance and single leg squat tasks. The objective of the study being to find if male PFP patients perform single leg squat and horizontal hop for distance with greater knee valgus angle than controls, and if the nature of the task changes the degree of knee valgus angle. 


\section{Materials and Methods}

\subsection{Subjects}

Forty-five asymptomatic male participants (control) involved in the study testing (age mean $25.2 \pm 3.98$ year, height mean $171.96 \pm 5.37 \mathrm{~cm}$, and weight mean $74.80 \pm 6.33 \mathrm{~kg}$ ). All subjects had no any history of anterior cruciate ligament (ACL) injury or other knee pathology, lower limb pathology, lower limb fractures, lower limb surgeries, and had no sever injuries for 3 months prior to the data collection. Twenty male patients with unilateral patellofemoral pain (age mean $25 \pm 3.9$ year, height mean $172.1 \pm 4.93 \mathrm{~cm}$, and weight mean $73.6 \pm 6.44$ $\mathrm{kg}$ ) were recruited from hospital clinics, who were the symptomatic comparison group. These patients have been already examined by experienced musculoskeletal doctors to establish that they have met the required inclusion and exclusion criteria mentioned in Table 1 [17], and they are only having unilateral knee pain. To minimize the risk of any symptom aggravation with testing, participants with relatively mild symptoms (pain is less than 8 out of 10 on a $10 \mathrm{~cm}$ visual pain scale, whereas 0 equals no pain and 10 worse perceivable pain) were selected to take part in the study. All participants were recreational athletes participated at least 3 hours of sporting activity per week. A written informed consent was obtained from all subjects and the project was approved by Najran University Research Ethics Committee with approval number (10 - 05-01 - 2020 $\mathrm{EC})$.

\subsection{Procedures}

\subsubsection{Frontal Plane Projection Angle (Video Capture)}

The frontal plane projection angle (FPPA) was assessed using a single camera, capturing at a standard sampling frequency of $30 \mathrm{fps}$, positioned on a tripod at a height of $80 \mathrm{~cm}$ from the floor to the middle of the lens, and $2.5 \mathrm{~m}$ away from an $\mathrm{X}$-shaped marker which was placed as a reference for the central point on the floor. The zoom lens of the video camera was set at a standard 1x optical zoom throughout all trials in order to standardize the camera position between participants. The reason behind placing the camera on a tripod at a height of $80 \mathrm{~cm}$ and $2.5 \mathrm{~m}$ away is to ensure that the video included the lower limbs, trunk, and shoulders of the participants with different heights. Each participant was filmed before starting any of the individual tests using a calibration frame $(1 \mathrm{~m} \times 1 \mathrm{~m})$ for five seconds. The calibration distance was set $2.5 \mathrm{~m}$ away from a camera (frontal plane) just above the $\mathrm{X}$ mark which was placed on the floor. This calibration was used for data analysis.

In order to examine the FPPA, three markers were placed directly on the participants' skin before starting the test using a black marker on the following points:

1) Anterior superior iliac spine (ASIS).

2) Middle of the tibiofemoral joint (not middle of the patella).

3) The middle of the ankle mortise anatomical landmark. 
Table 1. Inclusion exclusion criteria for patellofemoral pain patient group (Herrington and Al-Shehri 2007).

Inclusion criteria

- Symptoms of anterior knee pain for at least 1 month

- Average pain level of 3 or more on a $10-\mathrm{cm}$ visual analog scale during stepping up and down of a $30 \mathrm{~cm}$ high bench

- Anterior or retropatellar knee pain on at least 2 of the following activities: prolonged sitting, climbing stairs, squatting, running, kneeling, and hopping/ jumping

- Presence of two of the following clinical criteria on assessment: pain during apprehension test, pain during the patellar compression test, and crepitation during the compression test

Exclusion criteria

- Previous knee surgery or arthritis

- History of patellar dislocation or subluxation, or ligament laxity

- Patellar tendon pathology or chondral damage

- Spinal referred pain

- History of other abnormalities such as leg length inequalities (N $2 \mathrm{~cm})$

- Medication as a part of the treatment

- Previous physical therapy or acupuncture treatment for the knee within the previous 30 days

All markers were placed by the same experimenter, and the midpoints were determined using a standard tape measure (Figure 1). Participants were asked to perform 3 test trials for all tasks onto their right (dominant in all cases) leg for the control group and both symptomatic and asymptomatic limbs for the PFP group. The reason why the control group has to be right dominant in all cases is that to standardize the test protocol and to make sure that there were no variations might occur when conducting the tests between both limbs (right and left). Moreover, to make sure that the reference values provided in the current study can be generalized to one limb (right) only. The analysis of the FPPA was undertaken in Quintic Biomechanics Software (v21, Quintic, Sutton Coldfield, UK) where FPPA was taken at the maximum knee flexion angle after landing from hop and squat (defined as the lowest point the pelvis reached). After recording the results from the three successful trials for each participant, the mean value over the three trials was calculated and reported. The test-retest reliability of the following tests for evaluating an individual's repeatability of performance of the knee valgus has been reported previously and shows that are reliable tests [12] [18].

1) Single-Leg Squat Task

Participants were instructed to stand on one leg, keep the other limb off the floor, with hands crossed behind their trunk in order to allow all markers to be visible. They were asked to squat down to $45^{\circ}$ (estimated visually) but not greater than $60^{\circ}$, and then return to a normal position without losing their balance. During practice trials (from three to five trials maximum), knee flexion angle was checked using a standard goniometer (Gaiam-Pro) then observed by the examiner throughout all trials. There was also an electronic counter used for each trial over five second period in which the first count starts the movement, 


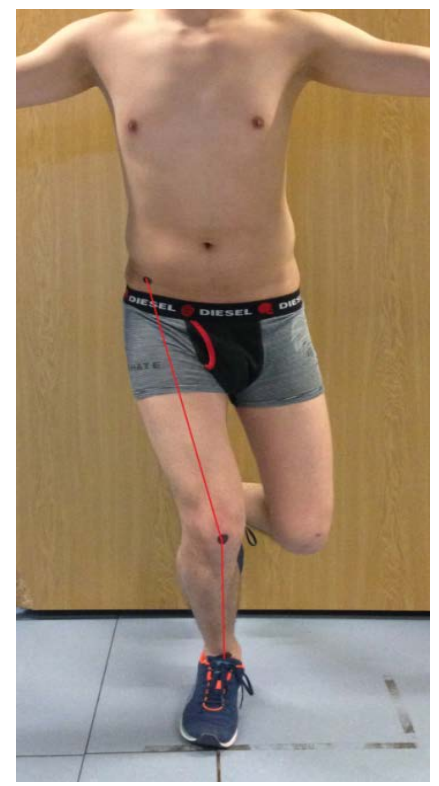

Figure 1. Anatomical marker placement to determine the FPPA.

the third shows the lowest point of the squat and the fifth shows the end. In order to control the degree of lower limb rotation during squatting, the participants were instructed to place their foot on the X-shaped marker, which is placed on the floor, with their foot pointing straight ahead. Acceptable trials were when participants maintained balance and squatted to the desired depth of approximately $45^{\circ}$ of the knee joint. The control group applied this task on their dominant (right dominant in all cases) leg, while the PFP group applied the task on both legs. While carrying out the task perceived pain was recorded (scored after completion) by the subject marking perceived pain level on a $10 \mathrm{~cm}$ visual pain scale (0 equals no pain, 10 worse perceivable pain).

\section{2) Single-Leg Hop Landing Task}

The FPPA was assessed during the single-leg horizontal hop for distance test. Participants were asked to perform a unilateral horizontal hop for distance task as far as possible, and land with complete stabilization within the area of the $\mathrm{X}$-shaped marker which was placed on the floor $2.5 \mathrm{~m}$ far away from a camera (the hop was applied after adjusting the starting point). The participants hopped to the X-shaped marker (or nearby) from a starting point based on their individual hop distance achieved during the practice trials (from three to five trials maximum), to ensure that the landing was at a point $\pm 30 \mathrm{~cm}$ from the $\mathrm{X}$-shaped marker, to accommodate the calibration.

After landing, the participants were free to move their arms as required and to help with balance following landing. Unsuccessful attempts were when the participant hopped and touched the ground with their non-weight bearing leg during landing, or failed to hop within the limited marked distance. The participants needed to land with their foot in line with the camera to ensure that the appropriate calculation of the FPPA was achieved. If the individual landed with 
their foot too abducted or adducted this trial was repeated as this will affect the measurement of the FPPA. The control group applied this task on their dominant (right dominant in all cases) leg, while the PFP group applied the task on both legs. While carrying out the test perceived pain was recorded (scored after completion) by the subject marking perceived pain level on a $10 \mathrm{~cm}$ visual pain scale ( 0 equals no pain, 10 worse perceivable pain).

\subsection{Data Analysis}

All statistical analysis was conducted using SPSS for Windows version 25 (SPSS Inc., Chicago, IL). The relationship between knee valgus angle during single leg squat and single leg horizontal hop for distance for both controls and PFP patients was analyzed using factorial ANOVA two factors; task (single leg squat and single leg horizontal hop for distance) and condition (control, PFP or uninjured). Paired t-tests were used to evaluate specific differences within conditions and student t-test between group comparison with the Bonferroni correction ( $\alpha$ $=0.0125)$. The significance $\mathrm{p}$-value was set at 0.05 .

\section{Results}

The mean, standard deviation, and the range of the values for the asymptomatic control group and PTP group for both tests the single leg squat and single leg horizontal hop for distance were as shown in Table 2. There is increase in knee pain reported by the subjects who participated in the current study, with more pain is seen when undertaking hop landing tasks. Therefore, it is shown that increasing knee valgus angle during loaded taskes will significantly increase the knee pain. The numeric rating pain score for all limbs including all tasks was shown in Table 3.

Table 2. The mean, standard deviation, and the range of values for both groups.

\begin{tabular}{|c|c|c|c|c|c|c|c|c|}
\hline \multirow{2}{*}{ Group/Test } & \multicolumn{3}{|c|}{ Single Leg Squat } & \multicolumn{5}{|c|}{ Single Leg Horizontal Hop for Distance } \\
\hline & Mean & $\mathrm{SD}$ & Range & Mean & SD & Range & Cohen's d & $\mathrm{P}$-value \\
\hline PFP Group Asymptomatic Knee & $9.80^{\circ}$ & $1.37^{\circ}$ & $7.1^{\circ}-11.9^{\circ}$ & $13.72^{\circ}$ & $1.53^{\circ}$ & $10.9^{\circ}-16.4^{\circ}$ & -2.70 & $0.001^{\star}$ \\
\hline PFP Group Symptomatic Knee & $15.04^{\circ}$ & $1.67^{\circ}$ & $12.3^{\circ}-17.3^{\circ}$ & $19.17^{\circ}$ & $1.74^{\circ}$ & $16.3^{\circ}-22.7^{\circ}$ & -2.42 & $0.001^{\star}$ \\
\hline
\end{tabular}

$\mathrm{SD}=$ Standard Deviation, ${ }^{\circ}=$ Degree, ${ }^{*}$ Significant difference between tasks $(\mathrm{p}<0.05)$.

Table 3. Visual pain rating scale score for all tasks (mean \pm standard deviation).

\begin{tabular}{|c|c|c|c|c|c|c|}
\hline \multirow{2}{*}{ Group/Test } & \multicolumn{2}{|c|}{ Single Leg Squat } & \multicolumn{4}{|c|}{ Single Leg Horizontal Hop for Distance } \\
\hline & Mean & $\mathrm{SD}$ & Mean & $\mathrm{SD}$ & Cohen's d & $\mathrm{P}$-value \\
\hline PFP Group Asymptomatic Knee & 0.85 & 0.59 & 1.15 & 0.88 & -0.40 & $0.266^{*}$ \\
\hline PFP Group Symptomatic Knee & 4.50 & 0.83 & 6.00 & 0.65 & -2.01 & $0.681^{*}$ \\
\hline
\end{tabular}

$\mathrm{SD}=$ Standard Deviation, ${ }^{*}$ No significant difference between tasks $(\mathrm{p}>0.05)$. 
No significant interaction was shown between factors using factorial ANOVA $(\mathrm{P}=0.65,95 \% \mathrm{CI}[0.58-0.72])$. There was a significant effect for both tasks $(\mathrm{P}=$ $0.006,95 \% \mathrm{CI}[0.003-0.008])$, and for conditions $(\mathrm{P}=0.001,95 \% \mathrm{CI}[0.0005-$ $0.0009])$. Paired t-tests reported among the control knees $(P=0.002)$ with the mean $-4.67 \pm 2.26$, injured knees $(\mathrm{P}=0.001)$ with the mean $-4.13 \pm 2.45$, and uninjured knees $(P=0.001)$ with the mean $-3.92 \pm 2.03$, that there was a significant differences in FPPA between tasks. It was also found a significant difference between FPPA on single leg squat task between the control and injured knee $(P=0.0001$, with the mean $-8.62 \pm 2.31)$, the injured and uninjured knee $(\mathrm{P}=0.002$, with the mean $5.24 \pm 1.61)$, and between the control and uninjured $\operatorname{limb}(\mathrm{P}=0.007$, with the mean $-3.38 \pm 1.45)$. For single leg horizontal hop for distance FPPA there was a significant difference between the control and injured knee $(P=0.0003$, with the mean $-7.67 \pm 2.48)$, the injured and uninjured knee $(\mathrm{P}=0.001$, with the mean $5.45 \pm 1.89)$, and between the control and uninjured $\operatorname{limb}(\mathrm{P}=0.009$, with the mean $-2.22 \pm 2.27)$.

\section{Discussion}

The aim of the study was to investigate if male PFP patients single leg squat and horizontal hop for distance with greater knee valgus angle than controls, and if the nature of the different task changes the degree of knee valgus angle. Current study showed patients with unilateral PFP had significantly greater knee valgus angles than either their asymptomatic limb or asymptomatic controls when undertaking unilateral loading tasks.

This study found that patients with PFP showed significantly greater FPPA during single leg squats and single leg horizontal hop for distance than their contralateral asymptomatic limb or controls. Moreover, FPPA also showed to increase between tasks. Few previous studies have evaluated the effect of different tasks on PFP patient's individual performance. Lee [10] used 2-D motion analysis to assess FPPA in a unilateral single leg squat and single leg land tasks in patients with PFP and controls (females only). The main outcome in his study was to investigated the changes in knee valgus in PFP patients and controls across the tasks of single leg squat and single leg land. Lee [10] found that patients with PFP reported significantly greater FPPA during single leg squats and single leg land than their contralateral asymptomatic limb or controls. In line with this, our study demonstrated significant increases in knee FPPA between our tasks (single leg squats and single leg horizontal hop for distance), with the knee valgus increasing with the increased load to lower limbs. This finding is similar to what was reported by Lee [10] although we used in our study a horizontal hop land task and he used a land from a $30 \mathrm{~cm}$ step, similar outcomes have been noted.

In addition, Willson and Davis [4] used three-dimensional (3-D) motion analysis to evaluate changes in knee angle in PFP patients across the tasks of single leg squat, running, and single leg hopping. They reported that the PFP pa- 
tients have significant greater knee motion, however across these tasks the magnitude of that motion did not change. This was also similar to our findings, but the differences between our study and that of Willson and Davis [4] might be because of the differences in load in the respective tasks. They have patients hopping on the spot to an average height of $9.2 \mathrm{~cm}$, while in our study the subjects landing from a maximum horizontal hop for distance task. Maximum horizontal hop for distance appear to be more changeable and difficult than landing from a step, as it requires the limb to control the horizontal forces in addition to maintain the balance when landing. Therefore, this would potentially increase the stress on patellofemoral joint with the more load being focused on a specific contact area during landing [8]. This can be confirmed by the noticeable increase in knee pain reported by the PFP patients who participated in the current study (Table 3). This finding is in line with the work conducted by Salsich et al. [19] who found that increasing knee valgus angle during single leg squat will significantly increase the knee pain.

The mean FPPA between groups in a single leg squat task appeared to be lower in our study than Lee's [10] study (as this is the only similar task matched between the two studies). For the single leg squat task in our study we reported a mean of $6.96^{\circ}, 9.80^{\circ}, 15.04^{\circ}$ in the control, PFP asymptomatic knee, and PFP symptomatic knee, respectively. However, in Lee [10] study he reported a higher mean than our study of $8.4^{\circ}, 10^{\circ}, 16.8^{\circ}$ in the control, PFP asymptomatic knee, and PFP symptomatic knee, respectively. This variations and higher mean results in Lee's [10] study are expected as he got female participants in his study and we had males only, females on their nature have higher knee valgus angle than men as been reported by Nguyen \& Shultz [20] who found that the mean standing Q-angle in females is $13^{\circ}$ and it was higher about $4^{\circ}$ than what was reported by males $\left(9^{\circ}\right)$, thus, considering these variations reported in knee angle between gender in different tasks are normal. Regarding biomechanical models for male participants (controls) during standing task, the mean clinical measure reported for the Q-angle was $9.0^{\circ} \pm 4.1^{\circ}$ [20]. This is very close to the mean knee valgus angle reported in our study during squat task for the controls at $6.96^{\circ} \pm$ $1.56^{\circ}$, and for the asymptomatic knees for the PTP group at $9.80^{\circ} \pm 1.37^{\circ}$. However, it was different than (lower) to what was reported in our study during single leg hop land task by the controls $11.63^{\circ} \pm 1.84^{\circ}$ and asymptomatic knees for the PTP group $13.72^{\circ} \pm 1.53^{\circ}$. These findings would confirm that higher knee valgus angle is obtained during high force loaded exercises such as hop land tasks than lower force loaded exercises like squats.

Furthermore, it was found for single leg squat and single leg horizontal hop for distance FPPA tasks that there was a significant difference between the control and uninjured limb $(\mathrm{P}<0.009)$. The mean FPPA for the single leg squat was reported to be higher in uninjured limb than the control limb $9.80^{\circ}, 6.96^{\circ}$, respectively, and for the single leg horizontal hop for distance was $13.72^{\circ}$ for the uninjured limb and $11.63^{\circ}$ for the control limb. This means that the PFP patients 
are representing with higher knee valgus even with their sound limbs in comparison to the controls, hence they have already more abnormal pressure on their patellofemoral joint which may contribute to the presence of the pain on their injured limbs.

\section{Limitations}

There are possible limitations with using a 2-D for motion analysis. Although 2-D analysis was reported previously to be an accurate in measuring several tasks, the accuracy and magnitude of 3-D lower limb motion analysis during any movement cannot be fully replicated by 2-D FPPA applications. However, in the absence of the 3-D methods 2-D analysis still can provide a reliable and valid measures for lower limb kinematics [18]. Another limitation is that this study only included male participants, but the possible reason for this is because the previous study by Lee [10] was conducted on females only, hence we need to investigate if different gender will enhance the overall findings. Another limitation in the current study is that the number of participants in the control group far exceeds the symptomatic group and this may have affected the results. However, the control group was collected with almost similar characteristics to the symptomatic group to minimize any effect that may be presented with any variations of the age, height, and weight. Moreover, inter-limb testing for the symptomatic subjects was not performed and this is considered as another limitation of the current study.

\section{Conclusion}

Patients with PFP were represented with greater knee valgus angle on the injured limb (unilateral load) than what was found in either their sound asymptomatic limb or in the control group. More attention is needed to be taken for the knee valgus angle when treating/ rehabilitating patients or train athletes during any screening tasks such as a unilateral squatting, hop landing, or horizontal hop for distance task. If patients presented with higher knee valgus (more than $9.80^{\circ}$ according to our findings) treatment plan should be set. Treatment methods including patients' feedback in front of a mirror, stretching hip adductors, strengthening of hip abductors, and core strengthening exercises. If not corrected (the higher knee valgus), it may lead to more patellofemoral joint stress and ongoing pain.

\section{Acknowledgements}

The author is grateful to the Department of Physical Therapy at Najran University for their support. The author would like also to thank all patients and controls who participated in this project.

\section{Conflicts of Interest}

The author declares no conflicts of interest regarding the publication of this paper. 


\section{References}

[1] Witvrouw, E., Lysens, R., Bellemans, J., Cambier, D. and Vanderstraeten, G. (2000) Intrinsic Risk Factors for the Development of Anterior Knee Pain in an Athletic Population. A Two-Year Prospective Study. American Journal of Sports Medicine, 28, 480-489. https://doi.org/10.1177/03635465000280040701

[2] Blond, L. and Hansen, L. (1998) Patellofemoral Pain Syndrome in Athletes: A 5.7-Year Retrospective Follow-Up Study of 250 Athletes. Acta Orthopaedica, 64, 393-400.

[3] Utting, M., Davies, G. and Newman, J. (2005) Is Anterior Knee Pain a Predisposing Factor to Patellofemoral Osteoarthritis? Knee, 12, 362-365. https://doi.org/10.1016/j.knee.2004.12.006

[4] Willson, J. and Davis, I. (2008) Lower Extremity Mechanics of Females with and without Patellofemoral Pain across Activities with Progressively Greater Task Demands. Clinical Biomechanics, 23, 203-211.

https://doi.org/10.1016/j.clinbiomech.2007.08.025

[5] Wilson, N., Press, J., Koh, J., Hendrix, R. and Zhang, L. (2009) In Vivo Non Invasive Evaluation of Abnormal Patellar Tracking during Squatting in Patients with Patellofemoral Pain. The Journal of Bone and Joint Surgery. American Volume, 91, 558-566. https://doi.org/10.2106/JBJS.G.00572

[6] Tanamas, S., Teichtahl, A., Wluka, A., Wang, Y., Davies-Tuck, M., Urquhart, D., et al. (2012) The Associations between Indices of Patellofemoral Geometry and Knee Pain and Patella Cartilage Volume: A Cross-Sectional Study. BMC Musculoskeletal Disorders, 11, 87-95. https://doi.org/10.1186/1471-2474-11-87

[7] Noehren, B., Barrance, P., Pohl, M. and Davis, I. (2012) A Comparison of Tibiofemoral and Patellofemoral Alignment during a Neutral and Valgus Single Leg Squat: An MRI Study. Knee, 19, 380-386. https://doi.org/10.1016/j.knee.2011.05.012

[8] Lee, T., Morris, G. and Csintalan, R. (2003) The Influence of Tibial and Femoral Rotation on Patellofemoral Contact Area and Pressure. Journal of Orthopaedic \& Sports Physical Therapy, 33, 686-693. https://doi.org/10.2519/jospt.2003.33.11.686

[9] Hvid, I., Andersen, L. and Schmidt, H. (1981) Chondromalacia Patellae. The Relation to Abnormal Patellofemoral Joint Mechanics. Acta Orthopaedica Scandinavica, 52, 661-666. https://doi.org/10.3109/17453678108992164

[10] Herrington, L. (2014) Knee Valgus Angle during Single Leg Squat and Landing in Patellofemoral Pain Patients and Controls. The Knee, 21, 514-517. https://doi.org/10.1016/j.knee.2013.11.011

[11] Noyes, F.R., Barber-Westin, S.D., Fleckenstein, C., Walsh, C. and West, J. (2005) The Drop-Jump Screening Test Difference in Lower Limb Control by Gender and Effect of Neuromuscular Training in Female Athletes. American Journal of Sports Medicine, 33, 197-207. https://doi.org/10.1177/0363546504266484

[12] Ghulam, H.S., Herrington, L.C., Comfort, P. and Jones, R. (2015) Reliability of Hop Distance and Frontal-Plane Dynamic Knee Valgus Angle during Single-Leg Horizontal Hop Test. Journal of Athletic Enhancement, 4, 6.

https://doi.org/10.4172/2324-9080.1000218

[13] Decker, M.J., Torry, M.R., Wyland, D.J., Sterett, W.I. and Steadman, J.R. (2003) Gender Differences in Lower Extremity Kinematics, Kinetics and Energy Absorption during Landing. Clinical Biomechanics, 18, 662-669.

https://doi.org/10.1016/S0268-0033(03)00090-1

[14] Crossley, K., Zhang, W., Schache, A., Bryant, A. and Cowan, S. (2011) Performance 
on Single Leg Squat Task Indicates Hip Muscle Function. American Journal of Sports Medicine, 39, 866-873. https://doi.org/10.1177/0363546510395456

[15] Dierks, T., Manal, K., Hamill, J. and Davis, I. (2008) Proximal and Distal Influences on Hip and Knee Kinematics in Runners with Patellofemoral Pain during a Prolonged Run. Journal of Orthopaedic \& Sports Physical Therapy, 38, 448-456. https://doi.org/10.2519/jospt.2008.2490

[16] Myer, G., Ford, K., Barber Foss, K., Goodman, A., Ceasar, A., Rauh, M., et al. (2010) The Incidence and Potential Pathomechanics of Patellofemoral Pain in Female Athletes. Clinical Biomechanics, 25, 700-707. https://doi.org/10.1016/j.clinbiomech.2010.04.001

[17] Herrington, L. and Al-Shehri, A. (2007) A Controlled Trial of Open versus Closed Kinetic Chain Exercises for Patellofemoral Pain. Journal of Orthopaedic \& Sports Physical Therapy, 37, 155-160. https://doi.org/10.2519/jospt.2007.2433

[18] Munro, A., Herrington, L. and Carolan, M. (2012) Reliability of Two-Dimensional Video Assessment of Frontal Plane Dynamic Knee Valgus during Common Athletic Screening Tasks. Journal of Sport Rehabilitation, 21, 7-11. https://doi.org/10.1123/jsr.21.1.7

[19] Salsich, G., Graci, V. and Maxam, D. (2012) The Effects of Movement Pattern Modification on Lower Extremity Kinematics and Pain in Women with Patellofemoral Pain. Journal of Orthopaedic \& Sports Physical Therapy, 42, 1017-1024. https://doi.org/10.2519/jospt.2012.4231

[20] Nguyen, A.D. and Shultz, S.J. (2007) Sex Differences in Clinical Measures of Lower Extremity Alignment. Journal of Orthopaedic \& Sports Physical Therapy, 37, 389-398. https://doi.org/10.2519/jospt.2007.2487 